\title{
Die Fünfer-Symbolik der Tenggeresen in Ostjava, Indonesien
}

Die Tenggeresen leben zurückgezogen an den Abhängen des gleichnamigen Gebirges im Osten Javas. Von den ursprünglich ca. 40 Tenggerdörfern sind bis heute nur noch fünf völlig von islamischen Einflüssen frei geblieben und funktionieren als kulturelle und religiöse Einheiten. Ähnlich den Badui, einem anderen «Restvolk» im Westen Javas, wehren sich die Tenggeresen sehr bewußt gegen den missionarischen Druck der sie umgebenden islamisierten Bevölkerung und sind neben den genannten Badui die letzten Vertreter einer altjavanischen Kultur.

Im Herbst 1976 lebte ich während dreier Monate in einem dieser Dörfer und sammelte Material zum religiösen Selbstverständnis der Tenggeresen, das ich dann in meiner Lizentiatsarbeit unter dem Titel «Aspekte der Volksreligion der heutigen Tenggeresen in Ostjava» verarbeitete.

Die Basis dieser Volksreligion (Volksreligion im Gegensatz zur «offiziellen», hinduistisch geprägten Religion der Priester), oder anders gesagt, dieser gelebten Religion bildet ein Symbolsystem, das in der älteren Literatur - neuere gibt es kaum - nur andeutungsweise erwähnt wird.

Das mag daran liegen, daß es wohl allen Tenggeresen bewußt ist, nie aber explizit ausgedrückt wird. Ein älterer Tenggerese hat diese Haltung mir gegenüber einmal folgendermaßen formuliert: «Über die Form der Dinge sprechen wir, aber die Bedeutung, die wissen wir». Diese «Bedeutung» der Dinge beruht auf einem komplexen System von assoziativen Beziehungen, die sich in alle Bereiche der tenggeresischen Sach- und Geisteskultur erstrecken. Gemeinsames Merkmal aller Beziehungen und Angelpunkt des ganzen Systems ist die Zahl 5. Da sich die Beziehungen sonst aber nicht um ein Zentrum herum gruppieren, sondern ein enges, gleichmäßiges Netz bilden, drängt sich für eine Beschreibung kein Einstiegspunkt auf. Ich habe für diese Arbeit die Reihenfolge gewählt, in der ich während meines Aufenthaltes die einzelnen Zusammenhänge erkannt habe.

In der Vorstellung der Tenggeresen hängt alles, sowohl das Leben, als auch die Ordnung der Umwelt, vom ungestörten Gleichgewicht zwischen vier Lebensprinzipien ab. Dazu gehören die Erde, verehrt als Ibu Pêrtiwi, die Sonne (mata hari), sowie das männliche und das weibliche Prinzip. Diese vier bestehen aus zwei Zweiergruppen, wobei die letztere - männlich-weiblich - Leben erzeugt, das dann durch die Kräfte des ersten Paares - Sonne-Erde - erhalten wird. Diese Lebensmöglichkeit, oder Lebenskraft, die durch das Zusammenspiel dieser vier Kräfte garantiert wird, heißt «hurip». Hurip ist eine Art Lebensessenz - ich scheue mich, den Begriff des mana zu verwenden, obwohl es nahe liegen würde, weil hurip gewisse wichtige Eigenschaften des mana nicht besitzt; man kann seinen Anteil davon zum Beispiel nicht vergrößern, weder durch eine besondere Lebensweise, noch durch Töten und Essen von Tieren oder gar Menschen. Menschen, Tiere, aber auch Pflanzen und Steine besitzen ihren Anteil an hurip. Gusti, die tenggeresische Schöpfergottheit, hat auch sie geschaffen und wer sie nicht ehrt, der beleidigt den Schöpfergott selbst. Deshalb darf kein Tier getötet und keine Pflanze ausgerissen werden, es sei denn zur Erhaltung des eigenen Lebens.

Diese vier Kräfte oder Prinzipien, wie ich sie eher nennen möchte, stehen häufig in Beziehung zu fünf Farben: Rot und weiß werden sehr bildlich mit dem Menstruationsblut der Frau bzw. der Samenflüssigkeit des Mannes, also mit dem weiblichen und männlichen Anteil bei der Zeugung eines Menschen, in Verbindung gebracht. Gelb ist die Farbe der Sonne, sie kann in diesem System auch durch Wind (hawa) oder Luft ersetzt werden. Für die Tenggeresen ist der Wind nichts anderes als die spürbare Kraft der Sonne. Ibu Pertiwi, die Erde, schließlich ist schwarz. All diese Farben vereint, ergeben in der Vorstellung der Tenggeresen grün, die Farbe von hurip. Grün ist aber auch die Farbe der Fruchtbarkeit, die Lebensfarbe an sich.

Diese fünf Prinzipien haben in bezug auf den menschlichen Körper ihre spezifischen Sitze und Verehrungsorte. Rot, das weibliche Prinzip, sitzt im Ohr (pamirêng = Gehör), denn im Innern der Ohrmuschel wird das Rot des Körpers sichtbar. Das Wei $\beta$ der Augen (paningal $=$ Sehvermögen) ist der Sitz des männlichen Prinzips. Der Verehrungsort der Sonne ist die Nase (pangambu $=$ Geruchsinn), hier kann der Wind in den Körper eindringen. Außerdem enthält die Nase mit dem Gelb des Schleimes die Farbe der Sonne selbst. Da der Mensch die Nah-

Lic. phil. Barbara Lüem. Ethnologisches Seminar der Universität Basel. 
rungsmittel, die aus der Erde und dank deren Kraft wachsen, durch den Mund aufnimmt, wird der Sitz der Ibu Pêrtiwi selbst im Munde (pangucap = gesprochene Worte) gedacht. Hurip schließlich, als Zusammenfassung und Ergebnis der anderen vier, sitzt logischerweise im Zentrum des Körpers, im Nabel (pusêr). Eine weitere Verbindung besteht von den Farben zu den Himmelsrichtungen, indem jeder der vier Kardinalrichtungen eine Farbe zugeordnet wird:

\section{Osten zu weiß \\ Westen zu schwarz \\ Süden zu rot \\ Norden zu gelb}

Grün ist auch hier wieder das Zentrum, in diesem Falle das Individuum. Dieses Bild zeigt, so glaube ich, sehr deutlich, wie wichtig und eng die Beziehung und der Einklang zwischen dem Tenggeresen und seiner Umwelt sind.

Die Beziehung der zeitlichen Ebene liegt darin, daß auch den fünf Tagen der javanischen PasaranWoche Farben zugeordnet sind und zwar in den nachfolgenden Kombinationen:

kliwon zu grün
légi zu weiss
paing zu rot
pon zu schwarz
wagé zu gelb

Kliwon, als der wichtigste Tag der Woche - mit ihm beginnt sie -, trägt die Farbe grün.

Wenn wir nun alle diese Zweierbeziehungen nebeneinander stellen, so erhalten wir folgendes Schema, das nicht vollständig ist, sondern nur diejenigen $\mathrm{Be}-$ reiche erfaßt, in die ich im Laufe meines Aufenthaltes Einsicht gewinnen konnte:

\begin{tabular}{|l|l|l|l|l|}
\hline weiß & légi & Osten & männlich & Auge \\
\hline rot & paing & Süden & weiblich & Ohr \\
\hline schwarz & pon & Westen & Erde & Mund \\
\hline gelb & wagé & Norden & Sonne/Wind & Nase \\
\hline grün & kliwon & Zentrum & hurip & Nabel \\
\hline
\end{tabular}

Innerhalb einer der fünf waagrechten Spalten sind sämtliche Zweierbeziehungen möglich und werden von den Tenggeresen im täglichen Leben laufend hergestellt. Diese Beziehungen können sowohl positiv, als auch negativ sein; Augenkrankheiten beispielsweise können durch Auflegen von weißen Blütenblättern geheilt werden. Am Tage pon dagegen sollte man das Dorf möglichst nicht in Richtung Westen verlassen, weil das Schlechte, Kala, an diesem Tag im Westen droht. Im einen Falle dieser beiden
Beispiele wurde die Farbe mit dem Körperteil, das andere Mal der Wochentag mit der Himmelsrichtung in einen (positiven bzw. negativen) Zusammenhang gestellt.

Neben diesem an sich geschlossenen System werden die Farben auch Elementen zugeordnet: Rot gehört zu Feuer, gelb zur Sonne, schwarz zur Erde, weiß zu Wasser. Die beiden Systeme scheinen sich zu überschneiden. Ich stelle sie deshalb getrennt dar, weil das Rot des Feuers zum Beispiel niemals mit dem weiblichen Prinzip oder einer anderen, oben angeführten und mit "rot» assoziierten Erscheinung verbunden wird. Dasselbe gilt für die anderen drei, wobei hier Erde und Sonne nicht als Kraft oder Prinzip erscheinen, sondern als Materie, wobei Sonne auch hier mit Luft identisch ist.

Ihre graphische Darstellung findet diese breit gefächerte und in alle Lebensbereiche hineingreifende Symbolik im sogenannten Lampiran Buddha.

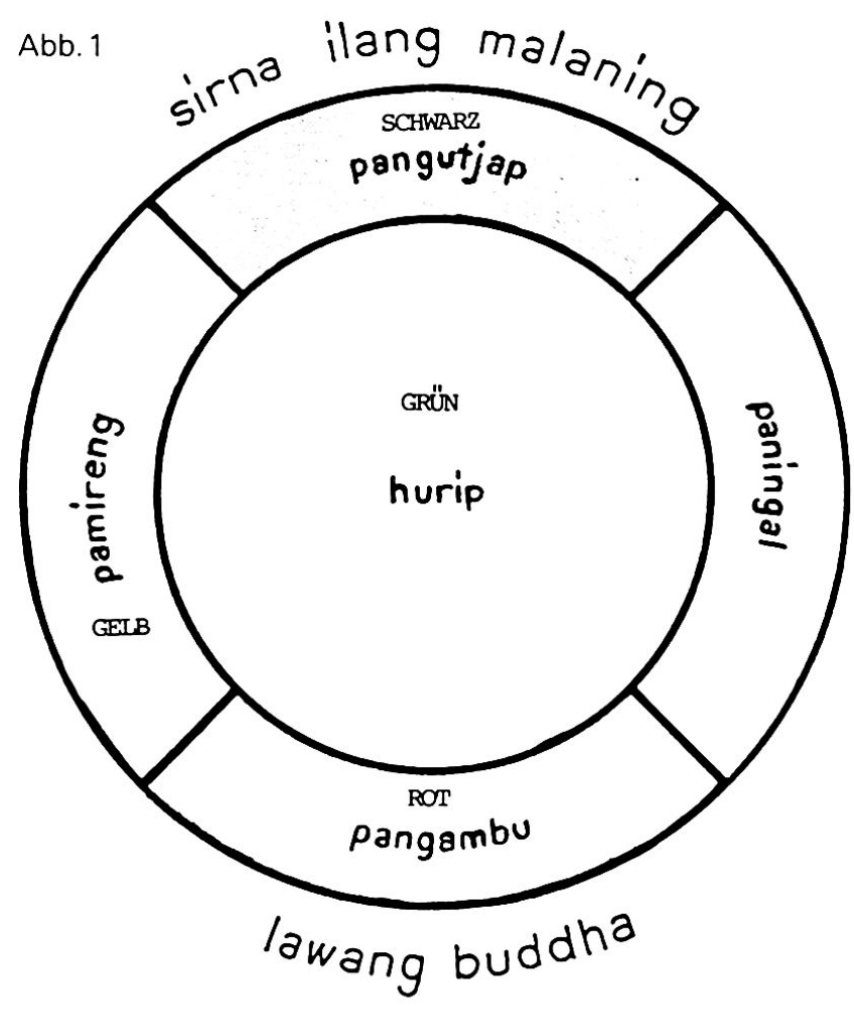


Übersetzung des Lampiran (= «Zeichnung») Buddha.

«sirna ilang malan ing lawang buddha»

$\begin{array}{ll}\text { sirna/ilang } & \text { verschwunden, zugrunde gegangen } \\ \text { mala }(n) & \text { das Böse, die Unreinheit } \\ \text { ing } & \text { in, bei, vor, durch } \\ \text { lawang } & \text { Türe, Pforte } \\ \text { pangucap } & \text { die gesprochenen Wörter } \\ \text { paningal } & \text { das Sehvermögen } \\ \text { pangambu } & \text { Geruch, das Riechvermögen } \\ \text { pamirèng } & \text { das Gehör } \\ \text { hurip } & \text { Lebenswasser, Leben }\end{array}$

"wêlas asih marang jiwa raga, bopo kuwoso, ibu pêrtiwi, bopo babu, sapada hurip, sato kéwan, tandur tuwuh"

$\begin{array}{ll}\begin{array}{l}\text { wêlas } \\ \text { asih }\end{array} & \text { Mitleid, Mitgefühl } \\ \text { marang } & \text { wohlwollend sein } \\ \text { jiwa } & \text { auf-zugehen; in bezug auf, gegenüber } \\ \text { raga } & \text { Lebensgeist } \\ \text { bopo } & \text { Körper } \\ \text { kuwoso } & \text { Vater } \\ \text { ibu } & \text { mächtig } \\ \text { pêrtiwi } & \text { Mutter } \\ \text { babu } & \text { Erde } \\ \text { sapada } & \text { Mutter } \\ \text { sato kéwan } & \text { gleich, all } \\ \text { tandur Tiere } & \text { das Gepflanzte, Reis } \\ \text { tuwuh } & \text { wachsen, gedeihen }\end{array}$

Der Satz würde dann ungefähr folgendes bedeuten:

«Zeige Mitleid/sei wohlwollend gegenüber: Geist und Körper (dem eigenen Geist)?), dem mächtigen Vater ( $=\langle$ Gott $\rangle$, der Mutter der Erde ( = «die Erdgöttin`, Vater und Mutter (Eltern`, allem, was lebt, den Tieren, allen gepflanzten Feldfrüchten».

Diese Zeichnung oder bildliche Darstellung des Symbolsystems auf Seite 84 ist meiner Meinung nach sehr jung, weil ich erstens nirgends in der Literatur einen Hinweis darauf finden konnte und es mir kaum wahrscheinlich vorkommt, daß alle früheren Autoren diese häufig vorkommende Darstellung übersehen haben, zumal das "Bild» von seinen Besitzern immer an gut sichtbaren Stellen aufgehängt oder hingestellt wird. Zweitens haben die Tenggeresen erst seit kurzem begonnen, traditionelles Wissen aufzuschreiben. Sie ziehen die mündliche Überlieferung auch heute vor und sind gegenüber schriftlichen Dokumenten bis heute mißtrauisch geblieben.

Eine Ausnahme bilden die mantra vom dukun, die in javanischer Schrift auf Lontarblättern fixiert sind.
Ein weiterer Hinweis darauf, wie unwichtig diese bildliche Darstellung der Symbolik für die Tenggeresen selbst ist, ist die Ungenauigkeit der Darstellung, obwohl die mündliche Erklärung einheitlich ist und also nicht unbedingt mit der Zeichnung übereinstimmt. Die Variation des Lampiran Buddha, die ich in dieser Arbeit wiedergebe, fand ich im Hause des dukun «meines» Dorfes. Obwohl er mich selbst darauf hinwies, daß die beiden Farben gelb und rot vertauscht seien, sieht er keinen Anlaß dazu, diesen Fehler zu verbessern. Hauptsache, er kennt die wahren Zusammenhänge. Ein weiterer Fehler ist die Zusammenstellung der Farben in bezug auf die Himmelsrichtungen. Gelb sollte der Windrose entsprechend oben sein, da es mit Norden assoziiert wird, weiß entsprechend links, schwarz rechts und rot als Süden unten. Dieser Mangel ist dem Besitzer des Lampiran Buddha, das er übrigens selbst gemacht hat, bewußt. In anderen Häusern habe ich Zeichnungen gesehen, die zwar den kosmischen Beziehungen gerecht werden, dafür aber andere Mängel aufwiesen. Ich habe absichtlich keine korrekte Ausführung, die ich übrigens auch nirgends gefunden habe, als Beispiel gewählt, obwohl dies meine Erklärungen etwas klarer und einfacher machen würde. Ich will aber damit der so bezeichnenden Divergenz zwischen Wissen und bildlicher Darstellung gerecht werden. Trotz dieser rein äußerlichen Schwierigkeiten, die sich aber für die Besitzer der Zeichnungen gar nicht stellen, ist die Absicht des Symbols deutlich: Es zeigt die Fünfer-Symbolik in ihren Zusammenhängen:

Die vier Farben der vier Lebenskräfte umschließen grün, die Farbe von hurip, der Lebensessenz, die durch das Zusammenspiel der anderen vier ermöglicht wird. Durch die graphische Anordnung und die Beschriftung wird außerdem der Bezug zum menschlichen Körper hergestellt. Nicht berücksichtigt wird der zeitliche Bezug.

Die Gebote, die unterhalb des eigentlichen Symbols notiert sind, bilden eine willkürliche Auswahl aus dem Gebot- und Verbotkodex der tenggeresischen Religion. Gerade weil die Auswahl willkürlich ist, will ich es bei einer Übersetzung belassen und lieber kurz auf die verschiedenen daneben existierenden Ge- und Verbotsammlungen eingehen.

Die erste Sammlung ist der sogenannte Dasar Agama, was frei übersetzt soviel wie «Grundlage der Religion" bedeutet. Seine sieben Punkte sind eine Art Katechismus, der früher den Kindern vom Vater gelehrt wurde. Heute hält der dukun innerhalb der Pfadfinderorganisation eine Art Religionsunterricht ab. Für diesen Unterricht hat er kürzlich alles aufgeschrieben, vorher beruhte das Ganze auf der mündlichen Überlieferung. Ich gebe die sieben Punkte hier übersetzt wieder: 


\section{Dasar Agama}

I. Alle Menschen leben zusammen auf einer Erde, über der das Schützende liegt. Alle zusammen müssen danach trachten, nur Gutes zu tun und die Ordnung der Dinge zu erhalten. Um diesen gemeinsamen Willen zu bekräftigen, ziehen am 14. Tag des Monats Kasada alle auf den Bromo, um zu opfern und sich zu reinigen. Wer diesem gemeinsamen Willen entgegenwirkt, muß diese Sünde Moho Kuwoso gegenüber ( $=G u s t i)$ verantworten.

II. Um Gutes zu tun und auf dem rechten Weg zu bleiben, müssen die Gedanken gut und rein sein. Man darf sich nicht zerstreuen, sondern sollte nur auf das Gute bedacht sein. Ein Weg dazu ist die häusliche Meditation (pamédi).

III. Um bei der Meditation erfolgreich zu sein, muß man Gusti darum bitten und sich vor ihm reinigen. Dies kann durch Meditation im sanggar pamujan geschehen.

IV. Die Menschen müssen das Gute im Zusammenhang mit der Verehrung von Gusti tun, denn Gusti gibt hurip, das wie Gusti selbst unsterblich ist. Was man Gutes in Verehrung für Gusti tut, das tut man auch für hurip. Das Gute bleibt damit ebenfalls unsterblich.

V. Der menschliche Körper hat neun Öffnungen (lobang sembilan), die man richtig verstehen und mit genügend Verehrung behandeln muß. Es sind dies:

1. lésan $=$ Mund

2. grono $=$ Nase (zwei Löcher)

3. nétro $=$ Auge (zwei Löcher)

4. talingan $=\mathrm{Ohr}$ (zwei Löcher)

5. wadi $=$ Geschlechtsöffnung

6. dubur $=$ After

Alle diese neun Öffnungen gleichen Wegen, die in den Körper hinein und aus ihm heraus führen. Man muß sehr sorgfältig darauf achten, daß nur Gutes darauf verkehrt, das heißt:

1. Man darf nichts Schlechtes oder Unfreundliches gegen irgendjemanden oder irgendetwas sagen.

2. Man soll schlechte Gerüche möglichst meiden («schlecht» im Sinne von übelriechend, aber auch im Sinne von «verboten»).

3. Man soll schlechte oder unfreundliche Reden nicht hören.

4. Man soll die Augen vor schlechten Dingen verschließen, da man sonst in Versuchung geführt werden könnte.

5. Man soll seine Geschlechtsöffnung nur am rechten Ort gebrauchen, das heißt man soll seinem Ehepartner treu sein.

6. Man darf heilige und öffentliche Plätze nicht durch seine Ausscheidungen verschmutzen.

Diese Gebote müssen befolgt werden, weil Gusti es so will.

VI. Alles, was die Menschen besitzen, kommt von
Gusti. Man muß damit zufrieden sein und darf nicht mehr verlangen. Der Dank dafür darf auf keinen Fall vergessen werden.

VII. Wer alle diese Gebote bis zum Tode gut befolgt, erhält im Himmel (swarga) einen guten Platz. Die Sünder erhalten keinen Himmelsplatz und müssen in den Wäldern als Geister (sétan) herumirren. Die guten Menschen, die sich in der Meditation so stark gereinigt haben, daß sie sich selbst wie vor einem Spiegel sehen, können später in einen menschlichen Körper zurückkehren. Diejenigen, die es nicht soweit gebracht haben, müssen im nächsten Leben als Hunde oder Wildschweine (babi hutan) weiterleben. Die zweite, populärere und wahrscheinlich auch später zusammengestellte Sammlung enthält weniger religiöse, als vielmehr moralische Ver- und Gebote. Sie stehen insofern mit der oben beschriebenen Symbolik im Zusammenhang, als daß sie in $2 \times 5$ Sätzen formuliert sind. Dieser Zusammenhang, der recht künstlich wirkt, wurde nicht von mir hergestellt, sondern ist den Leuten selbst sehr wichtig, denn diese Verbindung zur - ich glaube, man darf wohl sagen heiligen Zahl 5 gibt den zehn Sätzen einen gewissen religiösen und damit verbindlichen Charakter. Sie heißen nach ihren gemeinsamen Anfangsbuchstaben «Mo-limo» («limo» = 5) und «Wo-limo».

1. MALING $(=$ mencuri $=$ stehlen $)$. Dieses erste ist zugleich eines der wichtigsten Verbote, vor allem in bezug auf die Landwirtschaft. Die Felder liegen weit auseinander und oft einen Tagesmarsch vom Dorfe entfernt. Es wäre unmöglich, diese zu bewachen und die Feldfrüchte vor Dieben zu schützen. Da jeder Tenggerese in derselben Lage ist, kommen Diebstähle durch Tenggeresen höchst selten, wenn überhaupt, vor. Erst in jüngster Zeit wird dieses Verbot von Touristen übertreten.

2. MAIN $(=$ berjudi $=$ spielen). Jedes Spiel um Geld ist heute streng verboten. Ältere Männer mögen sich aber noch an das javanische Würfelspiel erinnern, das sie selbst häufig spielten. Wer das Spielen verboten hat, konnte ich nicht mehr feststellen. Heute ist Spielen um Geld sogar ein Scheidungsgrund.

3. MADAT (= Opium). Das Rauchen von Opium ist verboten, weil es die schlechten Gedanken fördert. Ausdrücklich wird auch das Verbrennen von Opium an Stelle von Weihrauch verboten, was ein kleines, aber sehr treffendes Licht auf die Erfindungsgabe der Tenggeresen wirft.

4. MINUM (= trinken). Dieses Verbot bezieht sich auf alkoholische Getränke. Mäßiges Trinken ist zwar erlaubt, aber die Gedanken müssen dabei klar bleiben. Es sind wiederum die Touristen, die den Verkauf von alkoholischen Getränken fördern.

5. MADON (= berzina $=$ Ehebruch). Dieses Gebot gilt vor allem für den Mann, denn die Tenggerfrau genießt eine besondere Stellung. So wohnt der Mann im Hause der Familie seiner Frau und ein Ehe- 
bruch wäre eine direkte Beleidigung der Gastfamilie und, in Anlehnung an die Ursprungsmythe, auch eine Beleidigung gegen Roro Antèng, die Stammesmutter der Tenggeresen.

Im Gegensatz zu den Mo-limo enthalten die Wo-limo nur Gebote, die jene fünf Dinge betreffen, die nach Meinung der Tenggeresen für das Leben jedes einzelnen und der ganzen Gemeinschaft am wichtigsten sind.

1. Waras ( $=$ sehat $=$ Sicherheit). Sicherheit ist hier im Sinne von materieller und moralischer Sicherheit gemeint. Sicherheit bedeutet aber auch Friede und Ordnung. Die Tenggeresen besitzen einen ausgeprägten Gemeinschaftssinn, was diese Sicherheit des einzelnen, des Dorfes und der ganzen Gemeinschaft betrifft. Sie soll gemeinsam erhalten werden.

2. Warêg $(=$ makan $=$ essen $)$. Gemeinsam sollte dafür gesorgt werden, daß alle genügend zu essen haben. Faulheit, aber auch Anhäufung von zu großen Vorräten ist verpönt.

3. Wutuh $(=$ sandang $=$ Tuch $)$. Jeder Tenggerese sollte genügend Stoff für einen sarong besitzen, der in dieser relativ kühlen Gegend das Universalkleidungsstück ist. Ich konnte während meines Aufenthaltes nicht weniger als elf verschiedene Tragarten, jede mit einer speziellen sprachlichen Bezeichnung, finden.

4. Wismo ( $=$ rumah $=$ Haus). Neben Nahrung und Kleidung ist das Haus für einen Tenggeresen absolut lebensnotwendig. Ein Haus besitzt neben seinen üblichen Funktionen einen großen sakralen Wert als Lebensbereich einer Familie.

5. Wasis $(=$ pintar $=$ Schlauheit $)$. Um genügend Sicherheit, Nahrung, Kleidung und ein Haus zu erhalten, braucht man eine gute Portion Schlauheit. Schlauheit ist hier nicht im schlechten Sinne gemeint, sondern bedeutet jene "pfiffige» Klugheit, die ich immer wieder beobachten konnte, und die für einen Tenggeresen eine erstrebenswerte Tugend ist.

\section{Die Fünfer-Symbolik im täglichen Leben}

Die vorangegangenen Beispiele zeigen, daß der normale Ablauf alles Lebenden vom ausbalancierten Zusammenspiel der fünf Prinzipien abhängt. Umgekehrt läßt eine Störung dieses normalen Ablaufs, wie zum Beispiel eine Krankheit oder Mißernten, auf eine entsprechende Störung im Kräftegleichgewicht der Prinzipien schließen, die, um die Ordnung wieder herzustellen, behoben werden muß. Das kann dadurch geschehen, daß man die Steine auf dem $p a-$ danyangan wieder zurechtrückt, wenn die "Störung» das Dorf betrifft, oder, im Falle von Krankheit, durch eine Behandlung oder ein Medikament, die irgendeine Verbindung zu den fünf Prinzipien aufweisen. Gegen Fieber, sakit panas, hilft zum Beispiel folgendes Rezept:
Weizenblatt (daun trigu, grün), gebratener Lauch (bawang apang, rot), ein farbloses Öl, das vor allem von den Chinesen verwendet wird (minyak kayu putih, weiß), Saft einer Zitrusfrucht (air jêruk bécèl, gelb) und Petrol (minyak tanah, schwarz) werden zu einem Brei verarbeitet. Der Patient wird damit eingestrichen und innerhalb weniger Stunden soll das Fieber sinken.

Gegen Kopfschmerzen hilft zerstoßener Gelbwurz (kunir) mit Öl vermischt. Dieser Brei soll auf die Stirne gestrichen werden. Die Wirkung dieses Medikamentes liegt an der gelben Farbe. Gelb war nämlich auch die Flüßigkeit, die damals bei der Geburt des Patienten beim Durchtrennen der Nabelschnur austrat. Zu jenem Zeitpunkt aber war das Neugeborene noch rein und das Kräftegleichgewicht noch ungestört.

Es gibt unzählige solcher Medikamentenrezepte, die alle an eine Art Analogie-Magie denken lassen. Parallel zur Anwendung dieser Medikamente wird aber immer auch Gusti um seine Hilfe gebeten. Hat die Behandlung Erfolg, so erhält er (Gusti) ein Dankesopfer, andernfalls jedoch nicht.

Ein wenig anders gelagert liegt die Wirkung der "Amulette», die zum Schutz des Hauses und seiner Bewohner über jede Türe angebracht werden. Sie bestehen aus zwei Teilen: einem weißen, länglichen Säckchen, dem sarat, und einem im Mittelnerv halbierten Palmblatt, dem canur.

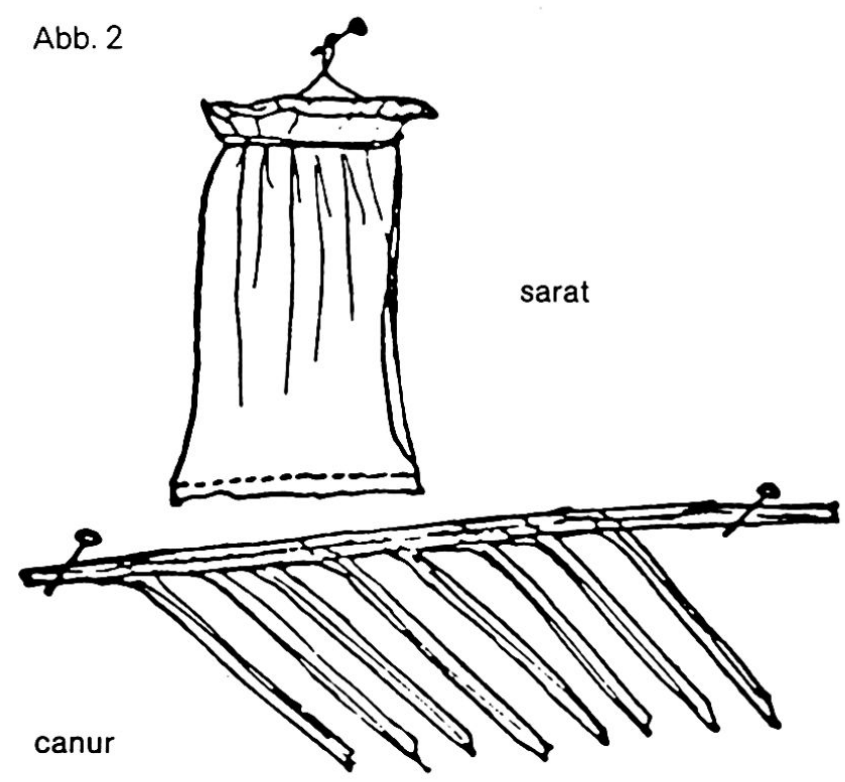

Der sarat muß aus fünf Bestandteilen zusammengesetzt sein, die farblich den fünf Prinzipien entsprechen müssen. Es gibt solche, die außerdem die Bestandteile des menschlichen Körpers darstellen. Ein solcher sarat besteht dann aus einer Nadel (jarum), die wegen der Kupferlegierung leicht grünspanig wird, aus einem Stück gelblich gewachsten Docht (bênang), aus einem Bausch rot gefärbter Watte ( $k a$ pas), aus einem Knäuel schwarzem Bindfaden (lawé) 
und aus dem weißen, die vier anderen umhüllenden Baumwollgewebe (kain putih). Die Nadel stellt die Seele und das Tuch die Haut dar. Der Docht soll die Adern, der Bindfaden die Eingeweide darstellen, und das Fleisch wird in der Watte gesehen.

Der canur seinerseits stellt einen menschlichen Körper dar, wobei der Blattnerv die Wirbelsäule sein soll und die fransenartig nach unten hängenden Blätter die Extremitäten und Geschlechtsteile. Dieses Amulett soll jeden daran hindern, die Türe zu durchschreiten, dessen Gedanken nicht ebenso rein sind, wie die vier sarat-Bestandteile in ihrem weißen Tuch. Dieses Hindernis besteht auch für übelwollende Geister oder Ahnen, die, ob sichtbar oder unsichtbar, Menschengestalt besitzen und den Gesetzen der fünf Prinzipien gehorchen müssen.

GORIS wurde um 1920 ein Gegenstand gezeigt, «... dat moeilijk juister te omschrijven valt dan als een groote worst, een reeks aaneengeregen saucijsjes» (1929: 222). GORIS fährt in seiner Beschreibung weiter:

«Het is een zeer lange lap goed geweest, waarin men met dun touw ruim twintig insnoeringen heeft aangebracht. In het bol uitstaande gedeelte van het doek, telkens tusschen twee insnoeringen in, werden verschillende voorwerpen gevonden, waarover zoo dadelijk... (S. 223)... Deze inhoud dan is in de meeste gevallen een opgerold lontarblad... Verder waren er kleine dunne metalen plaatjes in aanwezig, waarop in zeer onduidelijk schrift korte mantra's gekrast waren, o. a. herhaaldelijk het woord tigamaja. Ook was er een grooter metalen plaatje met een vrouwenfiguur erin gekrast. Een andere uitstulping bevatte een ris van elf Chineesche munten, de bekende kepengs. Merkwaardig was ook een stel van twee kleine potjes, een van wit aardewerk, en een in rood doek gewikkeld.» (S. 224)»

GORIS kann sich den Sinn dieser eingeschnürten Gegenstände nicht erklären. Er erwähnt, daß diese "Stoffwürste» als Schutz gegen Verletzungen umgehängt werden, bei einem Fechtspiel mit Bambusstöcken, womit wohl der sodoran gemeint ist. Die Zusammensetzung der Gegenstände durch fünf verschiedene Arten - Lontarblätter, Metallplättchen, Münzen und die beiden weiß gefärbten bzw. rot eingewickelten Töpfchen - lassen den Schluß sicher zu, daß wir es bei diesem «merkwaardige vondst» mit einem dem sarat ähnlichen Amulett zu tun haben, das seine schützende Kraft aus dem Zusammenspiel der fünf Prinzipien bezieht. Im Unterschied zu dem von mir beschriebenen sarat weist dieses "Amulett» allerdings einige hinduistische Elemente auf, die das zu Grunde liegende Prinzip überdecken.

In einen weiteren kulturhistorischen Zusammenhang gestellt, besitzt das oben beschriebene Assoziationssystem starke Anklänge an altjavanische Elemente.
J.PH.DUYVENDAK beschreibt in seinem kleinen Werk (1946: 116ff) das javanische Weltbild wie folgt:

«Het kosmisch verband, waarin de mens thuis hoort, bepaalt zijn geheele positie, in sociaal, economisch en religieus opzicht. Wie behoort bij een bepaalde maatschappelijke groep, behoort daardoor tevens tot een bepaalde religieuze eenheid, heeft deel aan bepaalde magische krachten. Deze voorstellingen leven thans nog onder de Javanen bij gedeelten voort, hoewel het systeem als zodanig niet meer wordt gekend. Men vindt brokstukken ervan nog terug in wichelpraktijken; in mystieke voorstellingen; in oude instellingen en gewoonten; in symboliek.» (S. 116)

Nach DUYVENDAK ist es der Sinn dieses Systems, eine sichere Ordnung in die Welt zu bringen. Durch eine Klassifikation werden Dinge zueinander in Beziehung gebracht, die von sich aus nichts miteinander zu tun haben. DUYVENDAK stellt ein Beispiel javanischer Klassifikationen schematisch dar (Seite 89).

Ein Vergleich mit dem Schema, das ich auf Seite 84 aufgezeichnet habe, ergibt eine weitgehende Übereinstimmung, die den Schluß sicher zuläßt, daß DUYVENDAK sich irrt, wenn er glaubt, daß dieses System heute nur noch in Bruchstücken vorhanden sei. Für die islamisierten Javaner mag dies zutreffen, das «Weltbild» der Tenggeresen aber, um DUYVENDAKs Ausdruck weiter zu verwenden, ist noch heute durch und durch von diesen Klassifikationen geprägt.

Dies wird besonders dort deutlich, wo neue Errungenschaften und Erscheinungen offensichtlich in dieses uralte System eingepaßt werden. Ich denke dabei beispielsweise an die Gebots- und Verbotssammlungen der Wo-Limo und Mo-Limo. Die darin enthaltenen Normen, die zum Teil erst in jüngster Zeit überhaupt entstanden sind, wie zum Beispiel das Verbot, Alkohol zu trinken, werden sehr mühsam in fünf Sätzen formuliert. Die Übereinstimmung der Anfangsbuchstaben liegt keineswegs auf der Hand, sondern mußte konstruiert werden, was sich daran zeigt, daß Ausdrücke aus der javanischen Sprache und der Bahasa Indonesia gewählt worden sind, um den rein äußerlichen Zusammenhang der fünf Anfangsbuchstaben zu schaffen. Das auf der Fünfzahl aufgebaute Klassifikations- und Assoziationssystem ist noch heute so stark im Bewußtsein der Tenggeresen verankert, daß es auch so banale Erscheinungen, wie eben dieses Alkoholverbot, einzubauen vermag.

Ein weiteres Beispiel für die Aktualität des fünfgeteilten Weltbildes ist die Kleidung des légèn und des wong sêpuh, der beiden Helfer des dukun.

Der légèn oder légi-Junge wird als rechte, also östliche Hand des zentral stehenden dukuns gedacht. Er trägt, sobald er als Assistent des dukun an die Öf- 


\begin{tabular}{|l|l|l|l|l|}
\hline I & II & III & IV & V \\
\hline ost & süd & west & nord & Mitte \\
\hline weiß & rot & gelb & schwarz & vielfarbig \\
\hline Silber & Kupfer & Gold & Eisen & Mischung \\
\hline Légi & Paing & Pon & Wagé & Kliwon \\
\hline umfassend & gierig & protzig & wortkarg & redegewandt \\
\hline Bauer & Händler & Palmweinzapfer & Schlachter & Fürst \\
\hline Nahrung & Geld & Alkohol & Fleisch & Göttin Sri \\
\hline Garten & Gott Gana & schwach & gebrochen & \\
\hline pendapa & Moschee & Küche & Stall & Haus \\
\hline Wind & Berg & Unglück & Feuer & Erde \\
\hline Wasser & schnell & kränklich & Flamme & beständig \\
\hline kühlgünstig & Platzwechsel & & & \\
\hline
\end{tabular}

fentlichkeit tritt, ein weißes Hemd. Der wong sêpuh dagegen, dessen Hauptaufgabe das Verbrennen der pétra (Seelenpuppe) ist, muß ein schwarzes Hemd tragen. Als ich mich während einer Beerdigung nach der Bedeutung dieser Hemdfarben erkundigte, wurde mir die lakonische Erklärung gegeben, das Hemd des légèn sei weiß, weil er eben légèn heiße und der wong sêpuh müsse darum schwarz tragen, weil er die Seelenpuppen verbrenne. Erst durch das erweiterte Assoziationssystem von DUYVENDAK wurden mir diese Antworten verständlich: Die Attribute des légèn - «légi», "östlich» und "weiß» - stehen alle in der ersten Spalte des Schemas, gehören also zur selben Kategorie, ebenso wie die Attribute des wong sêpuh - «Feuer» bzw. «Flammen» und "schwarz»beide in der fünften Spalte zu finden sind.

Es ließen sich noch unzählige Beispiele anführen. Die wenigen genannten zeigen aber schon deutlich, daß das alte javanische Weltbild bei den Tenggeresen weitgehend ungebrochen weiter besteht.

Einer von vielen Gründen dafür, warum die hinduistisch-buddhistische Epoche, von der die javanische Hofkultur entscheidend geprägt wurde, in der Religion des tenggeresischen Volkes - auf die Religion des dukun komme ich weiter unten noch zu sprechen - kaum Spuren hinterlassen hat, ist wohl der, daß sich der Mahāyāna-Buddhismus, der in dieser Zeit höchstwahrscheinlich dominierend war, an einem ähnlichen System orientiert, das von den Tenggeresen, leicht modifiziert, ohne große Schwierigkeiten übernommen und ins eigene System eingebaut werden konnte, ohne daß dieses dadurch wesentlich geändert worden wäre. Im Gegensatz zum herkömmlichen Buddhismus stehen an der Spitze des Mahāyāna-Buddhismus nicht die sieben menschlichen Buddhas, sondern die fünf himmlischen, meditierenden Dhyāni-Buddhas. Sie werden mit bestimmten Himmelsrichtungen, Farben, Jahreszeiten, metaphysischen Begriffen, Geschmackssorten, Zauberformeln und Lautgruppen des indischen Alphabeths verknüpft. Auf diese Weise entstehen fünf Kategorien, die mit den altjavanischen zwar nicht ganz identisch sind, denen aber ein ähnliches gedankliches Prinzip zu Grunde liegt (cf KIRFEL 1959: 43). KIRFEL fügt als vergleichbares Beispiel zum Mahāyāna-Buddhismus die fünf Gott-Kaiser der alten Chinesen an, die sich ebenfalls nach fünf Himmelsrichtungen orientieren. Jedem einzelnen von ihnen entspricht außerdem je eine Farbe, ein Planet, ein Element, eine Geschmacksrichtung usw. Auch hier war also die «Welt» in fünf Kategorien gegliedert. KIRFEL schreibt zu dieser Übereinstimmung:

«Für diese magische Verknüpfung der Fünfzahl mit Makro- und Mikrokosmos, Raum und Zeit, Physis und Psyche, kurz den mannigfachsten Erscheinungen des Lebens, ließen sich noch weitere Beispiele aufführen ... Es kann nun kaum bezweifelt werden, daß die Suprematie der Fünfzahl im Verein mit der eigenartigen Farborientierung nichts zufälliges ist, sondern daß hier der Grundzug einer bestimmten Kultur symbolisch zum Ausdruck kommt, die im Prinzip damals Zentral- und Ostasien beherrschte ... in den Farben und den übrigen Begriffsgruppen spiegelt sich offenbar jene Kultur wieder.» (cf KIRFEL 1959: 44). 
Auch wenn ich KIRFELs Ansicht über eine zentralund ostasiatische Einheitskultur nicht teile, so meine ich doch, daß der Buddhismus, als er mit seinem Einfluß den Tengger erreichte, nicht auf eine ihm völlig entgegengesetzte Kulturform stieß, sondern vertraute Grundzüge vorfand. Durch diese Ähnlichkeit wurde eine eigentliche Kraftprobe unnötig, und die bestehende und die neue Religionsform beeinflußten sich zwar gegenseitig, liefen im wesentlichen aber getrennt nebeneinander her, weil sie sich gegenseitig akzeptieren und verstehen konnten.

Zu dieser Annahme bin ich gekommen, weil ich beim Studium der Literatur und während meines Aufenthaltes im Tengger den immer stärkeren Eindruck gewonnen habe, daß die hindu-buddhistischen Elemente beinahe ausschließlich im Einflußbereich des dukun erkennbar sind, das heißt auf Gemeinschaftsund Dorfebene, kaum aber in der Familie.

Hinduistische Stilelemente sind nur an den Kultgegenständen des dukun - dem Weihwasserbecher, der Gebetsglocke und der Opferschale - zu finden. Die hinduistischen Götternamen tauchen ihrerseits nur in den mantra der dukun auf. Die upacara, jene vom dukun ausgeführte Zeremonie, wirkt bei allen religiösen Festlichkeiten so, als ob sie später dazu gefügt worden wäre, das heißt, sie bildet einen in sich geschloßenen Teil des Festes, der keinen Einfluß auf dessen weiteren Verlauf hat.

KOHLBRUGGE weist als einziger Autor auf die isolierte Stellung der Priester innerhalb der tenggeresischen Gesellschaft hin und schreibt:

«Vor vier Jahrhunderten fiel das Reich Modjopahit vor dem Ansturm der Mohammedaner, vielleicht stammt das Geschlecht (der dukun) von einem der geflohenen modjopahitschen Fürsten und würde die Sage von der Flucht vor dem Islam nach der Zerstörung von Modjophahit hierdurch erklärt sein. Also nicht die Tênggêresen selbst, die damals das Gebirge schon lange bewohnten, sondern ihr Priestergeschlecht wäre dann von modjopahitischer Herkunft.» (1901: 93)

KOHLBRUGGE kommt zu diesem Schluß auf Grund genealogischer Untersuchungen der dukun-Fami- lien, die sich im Tengger nur gerade bis zum Zeitpunkt des Niederganges von Majapahit zurückverfolgen lassen.

Ich glaube, das in dieser Arbeit dargestellte Material zeigt deutlich, daß der Hindu-Buddhismus, dem die Tenggeresen anhängen sollen, nicht die Religion des ganzen Volkes ist. Falls die Annahme von KOHLBRUGGE zutrifft, so haben die einwandernden Fürsten aus Majapahit den Platz einheimischer Priester eingenommen. Darauf hin deuten die verschiedenen Aufgaben, die von den dukun außerhalb des religiösen Lebens übernommen werden, wie zum Beispiel die Rechtsprechung, die wohl auch früher im Aufgabenbereich eines Priesters gelegen hat. Bei gewissen wichtigen Festlichkeiten, wie Hochzeiten und Begräbnissen, dagegen, ist seine Rolle verschwindend klein; hier sind der légèn und der wong sêpuh die leitenden Persönlichkeiten. Höchstwahrscheinlich stammen beide - wong sêpuh und légèn - aus vorhinduistischer Zeit, weil den von ihnen geleiteten Zeremonien die hindu-buddhistischen Elemente fehlen.

Weil die Einwanderer aus Majapahit sich in eine bereits bestehende Institution eingliederten und sich nicht erst eine neue Stellung schaffen mußten, blieb ihr Einfluß auch weitgehend auf diese Institution beschränkt.

\section{Bibliographie}

DUYVENDAK, J. PH. (1946): Inleiding tot de Ethnologie van de Indische Archipel. Groningen/Batavia: Wolters.

GORIS, R. (1929): «En merkwardige vondst op den Tengger", in: Tijdschrift voor Indische Taal-, Landen Volkenkunde. 69.

KIRFEL, W. (1959): Symbolik des Buddhismus. Stuttgart: Hiersemann.

KOHLBRUGGE, J. H.F. (1901): «Die Tenggeresen», in: Bijdragen tot de Taal-, Land-en Volkenkunde. 53.

LÜEM, B. (1977): Aspekte der Volksreligion der heutigen Tenggeresen in Ostjava. Basel (Manuskript).

Das Geographische Institut der Universität Zürich trauert um

\section{Professor Dr. Karl Suter}

ausserordentlicher Professor 1958-1968

Seine wissenschaftliche Leistung und seine Tätigkeit als Dozent werden in einem Nachruf gewürdigt werden. 